\title{
Aliskiren in combination with valsartan exerts synergistic protective effects against ventricular remodeling after myocardial infarction in mice
}

\author{
Yasutomi Higashikuni ${ }^{1}$, Minoru Takaoka ${ }^{1}$, Hiroshi Iwata ${ }^{1}$, Kimie Tanaka ${ }^{1}$, Yasunobu Hirata ${ }^{1}$, \\ Ryozo Nagai ${ }^{1}$ and Masataka Sata ${ }^{2}$
}

The efficacy of aliskiren, a direct renin inhibitor, in ventricular remodeling after myocardial infarction (MI) compared with conventional renin-angiotensin system (RAS) inhibitors remains to be defined. This study was performed to examine the protective effects of aliskiren and its addition to valsartan, an angiotensin-II receptor blocker, against ventricular remodeling after MI. MI was induced in 8- to 12-week-old C57BL/6 mice by ligating the left anterior descending artery. At 3 days after MI, mice were divided into five groups and were treated with the following: (1) phosphate-buffered saline (PBS); (2) hydralazine ( $10 \mathrm{mg} \mathrm{kg}^{-1}$ day $\left.^{-1}\right)$; (3) valsartan $\left(8 \mathrm{mg} \mathrm{kg}^{-1}\right.$ day $\left.^{-1}\right)$; (4) aliskiren $\left(25 \mathrm{mg} \mathrm{kg}^{-1}\right.$ day $\left.^{-1}\right)$; and (5) combined aliskiren $\left(25 \mathrm{mg} \mathrm{kg}^{-1}\right.$ day $\left.^{-1}\right)$ and valsartan $\left(8 \mathrm{~g} \mathrm{~kg}^{-1} \mathrm{day}^{-1}\right)$. With these doses of drugs, blood pressure-lowering effects compared with the PBS group were similar among the treated groups in sham-operated mice. At 28 days after MI, echocardiographic, hemodynamic and histological assessments demonstrated that monotherapy with valsartan or aliskiren alone significantly and similarly ameliorated ventricular remodeling after MI compared with the PBS and the hydralazine groups. Combination therapy of valsartan and aliskiren more greatly improved ventricular remodeling after MI with enhancement of angiogenesis and greater attenuation of tissue oxidative stress and inflammation. Our results indicate that aliskiren can be an alternative to conventional RAS inhibitors in the treatment of post-MI patients. Moreover, the dual therapy of valsartan and aliskiren may be more beneficial than either monotherapy. Further clinical trials will be warranted to sufficiently assess the safety and the efficacy of the use of aliskiren in post-MI patients.

Hypertension Research (2012) 35, 62-69; doi:10.1038/hr.2011.136; published online 11 August 2011

Keywords: myocardial infarction; pharmacology; remodeling; renin-angiotensin system

\section{INTRODUCTION}

Ischemic heart disease including myocardial infarction (MI) is the major cause of death in industrial countries. ${ }^{1,2}$ MI frequently causes left ventricular dilatation associated with cardiomyocyte hypertrophy and interstitial fibrosis of the non-infarcted myocardium. These changes in the geometry of left ventricle (LV), referred to as ventricular remodeling, contribute to the development of cardiac dysfunction and heart failure, which are associated with increased morbidity and mortality. ${ }^{3-5}$ Therefore, a prevention of this adverse ventricular remodeling is one of the most important pharmacological targets in treating post-MI patients.

A great body of evidence has demonstrated that the increased renin-angiotensin system (RAS) activity has a key role in the development of ventricular remodeling after MI. ${ }^{6}$ Currently, angiotensin-converting enzyme inhibitors (ACEIs) and angiotensin receptor blockers (ARBs) have been proven to be effective in attenuating the progress of ventricular remodeling by inhibiting the increased RAS activity. ${ }^{7-10}$ However, ACEIs and ARBs induce a compensatory rise in renin and its downstream RAS components that may eventually overcome their RAS-blocking effect. ${ }^{11}$ Thus, they may not sufficiently inhibit the RAS and thereby antagonize disease progression. Accordingly, it is of critical importance to develop therapeutic strategies that will inhibit the RAS activity more effectively.

Aliskiren is the first in a new class of orally effective direct renin inhibitors (DRIs) approved for clinical treatment. ${ }^{12,13}$ Aliskiren blocks the RAS by inhibiting the rate-limiting step in the RAS cascade, and reduces synthesis of all subsequent components of the cascade. Therefore, aliskiren may offer an alternative to ACEIs or ARBs in the treatment of post-MI patients. In fact, aliskiren was reported to improve cardiac function and ventricular remodeling after MI independent of blood pressure (BP) in mice. ${ }^{14}$ However, it is unknown whether aliskiren is superior to conventional RAS inhibitors in the

${ }^{1}$ Department of Cardiovascular Medicine, University of Tokyo, Bunkyo-ku, Tokyo, Japan and ${ }^{2}$ Department of Cardiovascular Medicine, University of Tokushima, Tokushima-shi, Tokushima, Japan

Correspondence: Dr M Sata, Department of Cardiovascular Medicine, Institute of Health Biosciences, University of Tokushima Graduate School, 3-18-15 Kuramoto-cho, Tokushima 770-8503, Japan.

E-mail: sata@clin.med.tokushima-u.ac.jp

Received 19 April 2011; revised 21 May 2011; accepted 17 June 2011; published online 11 August 2011 
treatment of ventricular remodeling after MI. As angiotensin I can be produced in renin-independent manner through enzymes such as cathepsin D and tonin, ${ }^{15,16}$ even DRIs may not completely block the RAS activity.

As each one of RAS-blocking agents may not fully inhibit the RAS activity, it is suggested that combination of them can achieve more potent RAS suppression by mutual reinforcement, and thus, may result in better clinical outcomes in post-MI patients. Especially, the inhibition of the first rate-limiting step of angiotensin II production and the blockade of its receptor by combination of DRIs and ARBs may be theoretically a promising strategy. However, the efficacy of this strategy remains unclear. This study was performed to assess the efficacy of aliskiren and its addition to valsartan in post-MI outcomes.

\section{METHODS}

\section{Experimental model of MI}

This study was approved by the Animal Care and Use Committee of the University of Tokyo. All experiments were performed according to the 'Guide for the Care and Use of Laboratory Animals.' ${ }^{17}$ MI was induced in 8- to 12week-old male C57BL/6 mice (Japan SLC, Shizuoka, Japan) by a permanent left anterior descending artery ligation as described previously with minor modification. ${ }^{18}$ Briefly, mice were anesthetized by intraperitoneal injection of sodium pentobarbital ( $50 \mathrm{mg} \mathrm{kg}^{-1}$ of body weight). A 24-gauge polyethylene tube was inserted into the trachea and mechanical ventilation was provided by a rodent ventilator (Model SAR-830; CWE, Ardmore, PA, USA). The chest was opened by the second left intercostal incision and the heart was exposed. The left anterior descending artery was ligated by 7-0 nylon suture approximately $3 \mathrm{~mm}$ below the tip of the left atrial appendage, which was determined according to the survival rate after MI in the preliminary experiment. Sham animals were prepared identically without undergoing ligation of the left anterior descending artery.

At 3 days after the operation, mice were divided into five groups and were treated as the following: (1) phosphate-buffered saline (PBS) $(n=39)$; (2) hydralazine ( $10 \mathrm{mg} \mathrm{kg}^{-1}$ day $\left.^{-1}, n=31\right)$; (3) valsartan $\left(8 \mathrm{mg} \mathrm{kg}^{-1} \mathrm{day}^{-1}, n=37\right)$; (4) aliskiren ( $\left.25 \mathrm{mg} \mathrm{kg}^{-1} \mathrm{day}^{-1}, n=27\right)$; (5) combined valsartan $\left(8 \mathrm{mg} \mathrm{kg}^{-1} \mathrm{day}^{-1}\right)$ and aliskiren $\left(25 \mathrm{mg} \mathrm{kg}^{-1} \mathrm{day}^{-1}\right)(n=24)$. Valsartan and aliskiren were kindly gifted from Novartis (Basel, Switzerland). Hydralazine was purchased from Wako Pure Chemical Industries (Osaka, Japan). Valsartan, aliskiren and PBS were given to mice via osmotic minipump (ALZET mini-osmotic pump, DURECT, Cupertino, CA, USA). Hydralazine was orally given to mice with gavage, and osmotic minipumps were not implanted into hydralazine-treated mice. We chose the doses of the drugs according to the previous studies and our preliminary experiments. ${ }^{19,20}$ With these doses of drugs, BP-lowering effects compared with the PBS group were similar among the treated groups in shamoperated mice. We combined valsartan and aliskiren at the same doses as either monotherapy so that we could assess whether further addition of RAS-blocking agents to baseline medication is beneficial to organ protection without further BP-lowering effects. BP of conscious mice was measured with the tail-cuff method (Softron BP-98A; Softron, Tokyo, Japan).

\section{Echocardiographic and hemodynamic measurements}

Transthoracic echocardiographic study was performed under anesthesia with sodium pentobarbital before surgery, and at 3 and 28 days after MI with a dynamically focused $15 \mathrm{MHz}$ linear-array transducer (EnVisor M2540A; Philips Medical System, Best, The Netherlands) with a depth setting of $1.5 \mathrm{~cm}$. M-mode tracings were recorded from the short axis view at the papillary muscle level of the LV. For hemodynamic measurement, the right carotid artery was cannulated by the micro pressure transducers with an outer diameter of $0.42 \mathrm{~mm}$ (Samba 201 and Samba Preclin 420 LP; Samba Sensors AB, Vastra Frolunda, Sweden), which was then advanced into the LV. ${ }^{18}$ Pressure signals

Table 1 Hemodynamic and echocardiographic data on sham-operated mice

\begin{tabular}{|c|c|c|c|c|c|}
\hline Compounds & PBS & Hydralazine & Valsartan & Aliskiren & Combined \\
\hline \multicolumn{6}{|l|}{ Tail-cuff measurement of BP } \\
\hline$n$ & 6 & 6 & 6 & 6 & 6 \\
\hline \multicolumn{6}{|l|}{ Hemodynamic data } \\
\hline$n$ & 5 & 5 & 5 & 5 & 5 \\
\hline $\mathrm{dp} / \mathrm{dt}$ maximum, $\mathrm{mm} \mathrm{Hgs}^{-1}$ & $8622 \pm 400$ & $7422 \pm 295^{*}$ & $6923 \pm 405^{*}$ & $6691 \pm 526^{*}$ & $6703 \pm 226^{*}$ \\
\hline $\mathrm{dp} / \mathrm{dt}$ minimum, $\mathrm{mm} \mathrm{Hgs}^{-1}$ & $-6790 \pm 316$ & $-5215 \pm 357^{*}$ & $-5035 \pm 360^{*}$ & $-5069 \pm 301^{*}$ & $-5639 \pm 399 *$ \\
\hline \multicolumn{6}{|l|}{ Echocardiography } \\
\hline$n$ & 7 & 7 & 7 & 7 & 7 \\
\hline \multicolumn{6}{|l|}{ Baseline } \\
\hline LVPWth, mm & $0.687 \pm 0.005$ & $0.693 \pm 0.004$ & $0.691 \pm 0.003$ & $0.689 \pm 0.001$ & $0.691 \pm 0.003$ \\
\hline \multicolumn{6}{|l|}{ Day 28} \\
\hline LVEDD, mm & $3.600 \pm 0.011$ & $3.574 \pm 0.007$ & $3.600 \pm 0.004$ & $3.610 \pm 0.013$ & $3.587 \pm 0.006$ \\
\hline $\mathrm{EF}, \%$ & $63.7 \pm 0.7$ & $64.4 \pm 0.6$ & $64.3 \pm 0.5$ & $63.5 \pm 0.7$ & $65.3 \pm 0.6$ \\
\hline IVSth, mm & $0.686 \pm 0.002$ & $0.683 \pm 0.002$ & $0.684 \pm 0.002$ & $0.684 \pm 0.002$ & $0.684 \pm 0.002$ \\
\hline LVPWth, mm & $0.690 \pm 0.002$ & $0.684 \pm 0.002$ & $0.693 \pm 0.003$ & $0.687 \pm 0.003$ & $0.690 \pm 0.003$ \\
\hline
\end{tabular}

Abbreviations:BP, blood pressure; EF, ejection fraction; IVSth, interventricular septum thickness; LVEDP, left ventricular end-diastolic pressure; LVEDD, left ventricle end-diastolic diameter; LVPWth, left ventricle posterior wall thickness; PBS, phosphate-buffered saline.

${ }^{*} P<0.05$ vs. PBS. 
were recorded using a MacLab data acquisition system (Model 400 with chart v4.2 software; AD Instruments, Colorado Springs, CO, USA) with a sampling rate of $5000 \mathrm{~Hz}$. Heart rate was kept at approximately 320-370 beats per minute to minimize data deviation. ${ }^{18}$

\section{Histological analysis}

Hearts were weighed and fixed in methanol and cut into four transverse sections: apical, middle, upper and basal sections. Each part was embedded in paraffin, and sectioned at $5 \mu \mathrm{m}$ thickness. Specimens were stained with hematoxylin and eosin or sirius red dyes as described previously. ${ }^{21}$ Myocyte cross-sectional area (CSA) and collagen volume fraction (CVF) were determined by quantitative morphometry of tissue sections from the middle section of the LV in hematoxylin and eosin and sirius red staining, respectively. Twenty cardiomyocytes per slide were measured for myocyte CSA calculation. Three to five random fields were examined for CVF analysis (magnification, $\times 200$ ). Immunohistochemical staining was performed with an anti-CD31 antibody (MEC13.3; BD Pharmingen, San Diego, CA, USA). Antibody distribution was visualized by the avidin-biotin complex technique and Vector Red substrate (Vector Laboratories, Youngstown, OH, USA). Quantitative assessment of capillary density was performed in the peri-infarction area of the middle section of the heart, which was identified by anti-CD31 staining, respectively. Five random fields were examined, and density was expressed as the number per square millimeter (magnification, $\times 400$ ).

For infarct size analysis at 28 days after MI, the boundary lengths of the infarcted and non-infarcted epicardial and endocardial surfaces were traced with a planimeter digital image analyzing software (ImageJ, Bethesda, MD, USA) after sirius red staining. Infarct size was calculated as the sum of the endocardial and epicardial scar length divided by the sum of the LV endocardial and epicardial circumferences of all the sections. ${ }^{18}$

\section{RNA extraction and reverse transcriptase-PCR analysis}

Total RNA was isolated from the myocardial tissues with TRIzol Reagent (Invitrogen, Carlsbad, CA, USA). Reverse transcription was performed with $1 \mu \mathrm{g}$ of total RNA, random hexamer primers and moloney murine leukemia virus (MMLV) reverse transcriptase (ReverTraAce- $\alpha$; TOYOBO, Osaka, Japan). ${ }^{18}$ For quantitative assessment of gene expression levels, quantitative real-time PCR analysis was performed. Specific mRNAs were quantified by SYBR Green real-time PCR Master Mix (TOYOBO) in an ABI PRISM 7000 thermocycler (Applied Biosystems, Foster City, CA, USA) under standard manufacturer's conditions. Data are expressed in arbitrary units that were normalized by correction for the signal obtained in the same complementary DNA preparation for glyceraldehyde 3-phosphate dehydrogenase mRNA. The primers for real-time PCR were as follows: interleukin-6 (IL-6), 5'-ACAAC CACGGCCTTCCCTACTT- $3^{\prime}$ and 5'-CACGATTTCCCAGAGAACATGTG-3'; monocyte chemotactic protein-1, 5'-CCACTCACCTGCTGCTACTCAT- $3^{\prime}$ and $5^{\prime}$-TGGTGATCCTCTTGTAGCTCTCC- $3^{\prime}$; tumor necrosis factor- $\alpha, 5^{\prime}$-TCCC AGGTTCTCTTCAA- $3^{\prime}$ and $5^{\prime}$-GGTGAGGAGCACGTAGTCGG-3'; glyceraldehyde 3-phosphate dehydrogenase, 5'-ATGACAACTTTGTCAAGCTCATTT-3' and 5'-GGTCCACCACCCTGTTGCT- 3 '.

\section{Assessment of oxidative stress in murine heart}

For assessment of oxidative stress in murine hearts, lipid peroxidation (Lipid Peroxidation Assay Kit, Oxford Biomedical Research, Oxford, MI, USA) in the myocardial tissues was measured according to the manufacturer's recommendation..$^{18}$ In lipid peroxidation assay, we measured concentrations of malondialdehyde and 4-hydroxyalkenals.

\footnotetext{
Statistical analysis

Data are expressed as mean \pm s.e.m. Kaplan-Meier method and the log-rank test were used for comparison of survival. Multiple group comparison was performed by one-way analysis of variance followed by StudentNewman-Keuls post hoc test. Values of $P<0.05$ were considered statistically significant
}

\section{RESULTS}

Impact of drugs on BP and cardiac function in sham-operated mice Hemodynamic and echocardiographic data on sham-operated mice were shown in Table 1 . When we measured BP with the tail-cuff method at 28 days after the operation, BP-lowering effects of hydralazine, valsartan and aliskiren were similar among the three groups in sham-operated mice with our tested doses. Combination of valsartan and aliskiren did not lead to further BP lowering with the tested dose. At 28 days after sham operation, hemodynamic measurement was also performed by micro pressure transducers under anesthesia. Systolic BP measured by micro pressure transducers showed the same tendency with that measured by the tail-cuff method. Left ventricular end-diastolic pressure was similar among the five groups, whereas maximum and minimum $\mathrm{dp} / \mathrm{dt}$ values were reduced in the treated groups compared with the PBS group. Echocardiographic data revealed no significant differences in LV geometry and function among the five groups at baseline and 28 days after sham operation.

\section{Survival and macroscopic changes after MI}

Compared with PBS, aliskiren and the dual therapy significantly reduced mortality after MI, whereas hydralazine or valsartan alone failed to improve survival after MI (Figure 1a). The survival rate of the combination therapy group was higher than that of the aliskiren monotherapy group, although the difference was not statistically significant. Most of dead mice died of cardiac rupture in each group.
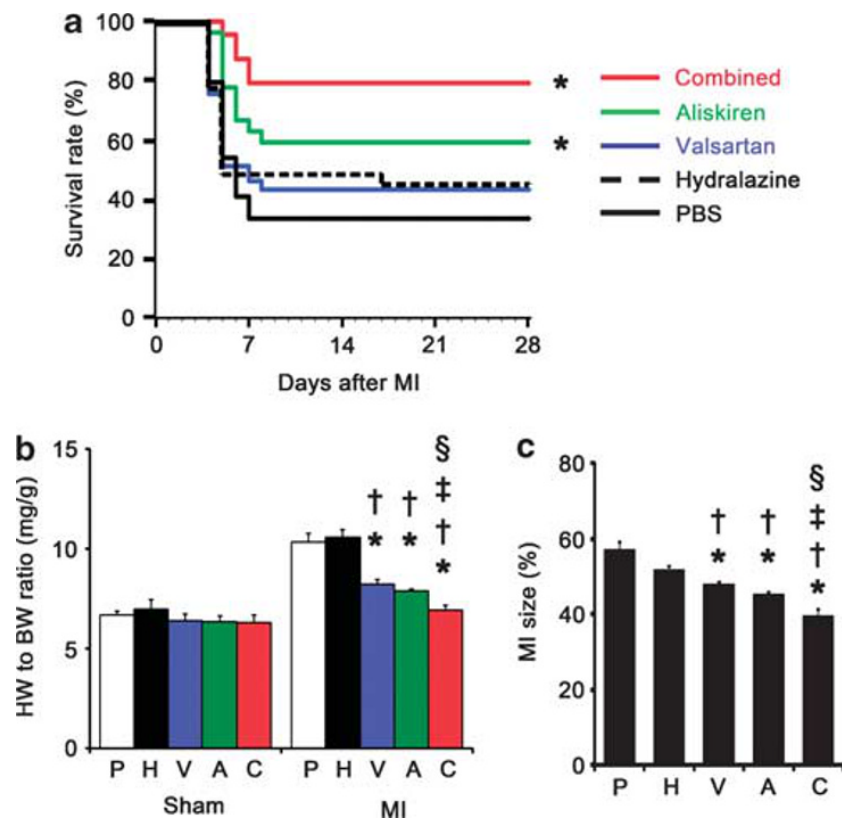

Figure 1 Survival and macroscopic changes after myocardial infarction (MI). (a) Survival curve after MI. Continuous black line, the phosphate-buffered saline (PBS) group ( $n=39$ ); dotted black line, the hydralazine group ( $n=31$ ); blue line, the valsartan monotherapy group $(n=37)$; green line, the aliskiren monotherapy group $(n=27)$; red line, the combination therapy group ( $n=24) .{ }^{*} P<0.05$ vs. the PBS group. (b) Heart weight (HW) to body weight (BW) ratios at 28 days after sham operation ( $n=5$ for each group) or MI ( $n=8$ for each group). (c) MI size at 28 days after the operation was assessed by sirius red staining ( $n=5$ for each group). P, the PBS group; $H$, the hydralazine group; $V$, the valsartan monotherapy group; $A$, the aliskiren monotherapy group; $C$, the combination therapy group. ${ }^{*} P<0.05$ vs. the PBS group; ${ }^{\dagger} P<0.05$ vs. the hydralazine group; ${ }^{\ddagger} P<0.05$ vs. the valsartan monotherapy group; ${ }^{\S} P<0.05$ vs. the aliskiren monotherapy group. 
The heart weight to body weight ratios at 28 days after sham operation were comparable among the five groups (Figure 1b). At 28 days after MI, valsartan or aliskiren alone significantly and comparably reduced the heart weight to body weight ratio compared with PBS, whereas hydralazine did not reduce it. The heart weight to body weight ratio of the combination therapy group was significantly lower than that of the monotherapy groups.

MI size at 28 days after the operation was also reduced by valsartan or aliskiren alone compared with PBS, although hydralazine failed to reduce it (Figure 1c). Furthermore, the dual therapy reduced MI size more than either monotherapy.

\section{Echocardiography and hemodynamics}

LV geometry and function, examined using echocardiography, were similar among the five groups before MI and at 3 days after MI (Table 2). Thus, echocardiographic data did not differ among the five groups before drug treatment. At 28 days after MI, hydralazine significantly reduced LV dilatation compared with PBS, although there were no statistically significant differences between the PBS and the hydralazine groups in wall thickness of the infarcted and the non-infarcted area and ejection fraction. Valsartan or aliskiren monotherapy significantly and similarly ameliorated LV dilatation, wall thinning of the infarcted area and wall thickening of the non-infarcted area, and improved ejection fraction compared with PBS and hydralazine. Moreover, the combination of valsartan and aliskiren improved LV geometry and function after MI more than either monotherapy.

Hemodynamic measurement showed that $\mathrm{BP}$, and maximum and minimum $\mathrm{dp} / \mathrm{dt}$ values were similar among the five groups at 28 days after MI (Table 2). Hydralazine did not reduce left ventricular enddiastolic pressure compared with PBS, whereas valsartan and aliskiren monotherapies significantly and comparably reduced left ventricular end-diastolic pressure. Left ventricular end-diastolic pressure of the combination therapy group was numerically, but not statistically, lower than that of the monotherapy groups.

\section{Myocyte CSA and CVF in the non-infarcted area}

In sham-operated mice, myocyte CSA and CVF of the non-infarcted area did not differ among the five groups at 28 days after the operation (Figure 2). In MI mice, myocyte CSA was reduced by hydralazine compared with PBS. Valsartan or aliskiren monotherapy further reduced myocyte CSA of the non-infarcted area than hydralazine. Moreover, the combination of valsartan and aliskiren improved cardiomyocyte hypertrophy more than either monotherapy.

Valsartan and aliskiren monotherapies improved cardiac fibrosis of the non-infarcted area compared with PBS, whereas there was no difference in CVF between the PBS and the hydralazine group. CVF of the combination therapy group tended to be lower than that of the valsartan or aliskiren monotherapy group, although the differences were not statistically significant with multiple comparison test.

Table 2 Echocardiographic and hemodynamic data on MI mice

\begin{tabular}{|c|c|c|c|c|c|}
\hline Compounds & PBS & Hydralazine & Valsartan & Aliskiren & Combined \\
\hline \multicolumn{6}{|l|}{ Echocardiography } \\
\hline$n$ & 10 & 10 & 10 & 10 & 10 \\
\hline \multicolumn{6}{|l|}{ Baseline } \\
\hline LVEDD, mm & $3.581 \pm 0.006$ & $3.580 \pm 0.005$ & $3.564 \pm 0.006$ & $3.567 \pm 0.014$ & $3.558 \pm 0.007$ \\
\hline$E F, \%$ & $64.1 \pm 0.4$ & $64.3 \pm 0.4$ & $63.3 \pm 0.3$ & $63.4 \pm 0.6$ & $64.8 \pm 0.6$ \\
\hline IVSth, mm & $0.683 \pm 0.002$ & $0.682 \pm 0.001$ & $0.686 \pm 0.002$ & $0.681 \pm 0.002$ & $0.688 \pm 0.003$ \\
\hline LVPWth, mm & $0.694 \pm 0.004$ & $0.691 \pm 0.004$ & $0.696 \pm 0.003$ & $0.689 \pm 0.003$ & $0.690 \pm 0.003$ \\
\hline \multicolumn{6}{|l|}{ Day 3} \\
\hline LVEDD, mm & $3.886 \pm 0.015$ & $3.881 \pm 0.010$ & $3.874 \pm 0.010$ & $3.864 \pm 0.013$ & $3.889 \pm 0.010$ \\
\hline$E F, \%$ & $31.4 \pm 0.7$ & $32.9 \pm 0.8$ & $32.5 \pm 1.0$ & $30.7 \pm 0.9$ & $31.2 \pm 0.7$ \\
\hline IVSth, mm & $0.627 \pm 0.004$ & $0.623 \pm 0.003$ & $0.619 \pm 0.005$ & $0.619 \pm 0.005$ & $0.613 \pm 0.003$ \\
\hline LVPWth, mm & $0.700 \pm 0.003$ & $0.697 \pm 0.003$ & $0.696 \pm 0.002$ & $0.695 \pm 0.005$ & $0.700 \pm 0.003$ \\
\hline \multicolumn{6}{|l|}{ Day 28} \\
\hline LVEDD, mm & $5.590 \pm 0.112$ & $5.253 \pm 0.054 *$ & $4.898 \pm 0.083 * \dagger$ & $4.678 \pm 0.074^{* \dagger}$ & $4.405 \pm 0.066^{*+1 \$}$ \\
\hline$E F, \%$ & $15.8 \pm 1.0$ & $17.8 \pm 1.1$ & $23.2 \pm 1.0^{* \dagger}$ & $24.1 \pm 1.6^{* \dagger}$ & $31.5 \pm 1.0 * \dagger+\S$ \\
\hline IVSth, mm & $0.469 \pm 0.011$ & $0.487 \pm 0.005$ & $0.556 \pm 0.006 * \dagger$ & $0.564 \pm 0.007^{* \dagger}$ & $0.587 \pm 0.005^{* *+\$}$ \\
\hline LVPWth, mm & $0.840 \pm 0.009$ & $0.835 \pm 0.007$ & $0.759 \pm 0.005^{* \dagger}$ & $0.748 \pm 0.005^{* \dagger}$ & $0.729 \pm 0.004 * *+\S$ \\
\hline \multicolumn{6}{|l|}{ Hemodynamic data on day 28} \\
\hline$n$ & 5 & 5 & 5 & 5 & 5 \\
\hline Systolic BP, mm Hg & $73.0 \pm 4.6$ & $69.7 \pm 4.6$ & $74.7 \pm 4.0$ & $72.4 \pm 1.0$ & $73.3 \pm 5.0$ \\
\hline LVEDP, $\mathrm{mm} \mathrm{Hg}$ & $10.75 \pm 1.97$ & $8.34 \pm 0.64$ & $4.05 \pm 0.77^{* \dagger}$ & $3.22 \pm 0.19^{* \dagger}$ & $2.14 \pm 0.27^{* \dagger}$ \\
\hline $\mathrm{dp} / \mathrm{dt}$ maximum, $\mathrm{mm} \mathrm{Hg} \mathrm{s}^{-1}$ & $5929 \pm 493$ & $5362 \pm 614$ & $6102 \pm 374$ & $6299 \pm 187$ & $5783 \pm 463$ \\
\hline $\mathrm{dp} / \mathrm{dt}$ minimum, $\mathrm{mm} \mathrm{Hg} \mathrm{s}^{-1}$ & $-4933 \pm 450$ & $-4605 \pm 303$ & $-4863 \pm 368$ & $-4755 \pm 177$ & $-4493 \pm 295$ \\
\hline
\end{tabular}

Abbreviations:BP, blood pressure; EF, ejection fraction; IVSth, interventricular septum thickness; LVEDD, left ventricle end-diastolic diameter; LVPWth, left ventricle posterior wall thickness; LVEDP, left ventricular end-diastolic pressure; MI, myocardial infarction; PBS, phosphate-buffered saline.

${ }^{\star} P<0.05$ vs. PBS.

${ }^{+} P<0.05$ vs. hydralazine

$P<0.05$ vs. valsartan.

$P<0.05$ vs. aliskiren. 
a
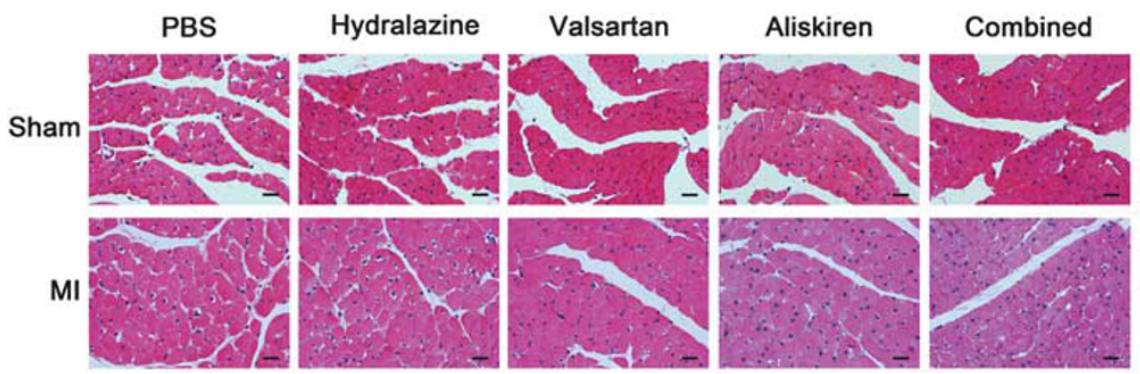

b

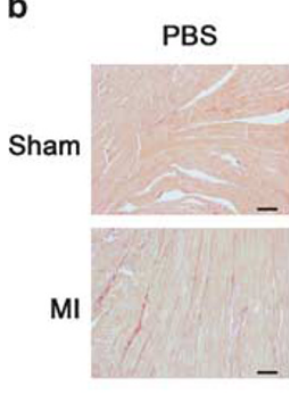

Hydralazine

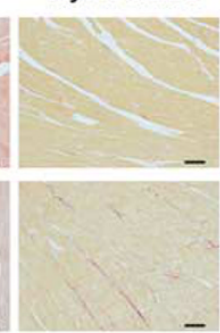

Valsartan

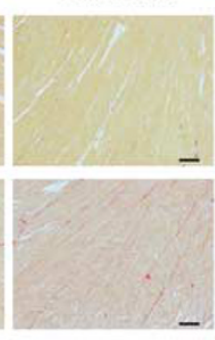

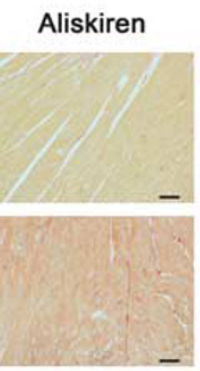

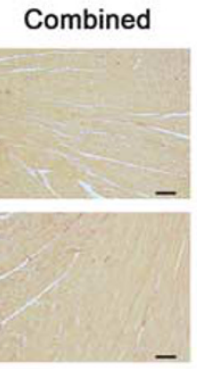

Myocyte CSA

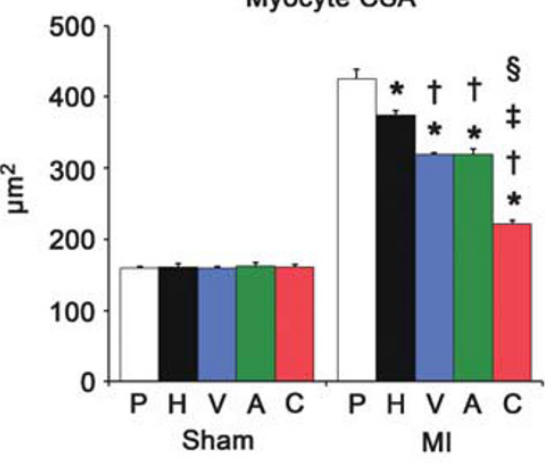

CVF

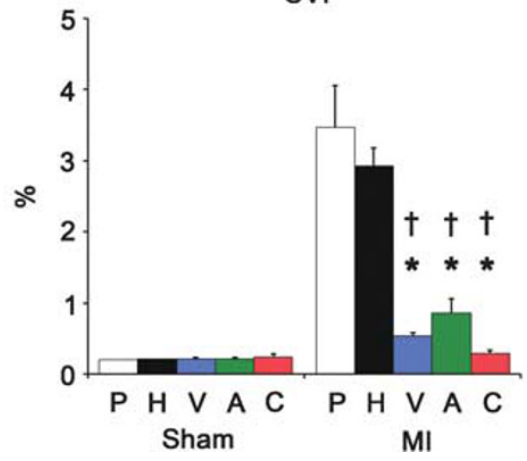

Figure 2 Cardiomyocyte hypertrophy and interstitial fibrosis in the non-infarcted area. (a) Myocyte cross-sectional area (CSA) and (b) collagen volume fraction (CVF) at 28 days after sham operation ( $n=3$ for each group) and myocardial infarction (MI) ( $n=5$ for each group) were assessed by hematoxylineosin and sirius red staining, respectively. $\mathrm{P}$, the phosphate-buffered saline (PBS) group; $\mathrm{H}$, the hydralazine group; $\mathrm{V}$, the valsartan monotherapy group; $\mathrm{A}$, the aliskiren monotherapy group; $C$, the combination therapy group. Scale bars $=20 \mu \mathrm{m}$ for (a) and $50 \mu \mathrm{m}$ for (b). ${ }^{*} P<0.05$ vs. the PBS group; ${ }^{\dagger} P<0.05$ vs. the hydralazine group; ${ }^{\ddagger} P<0.05$ vs. the valsartan monotherapy group; ${ }^{\circledR} P<0.05$ vs. the aliskiren monotherapy group.
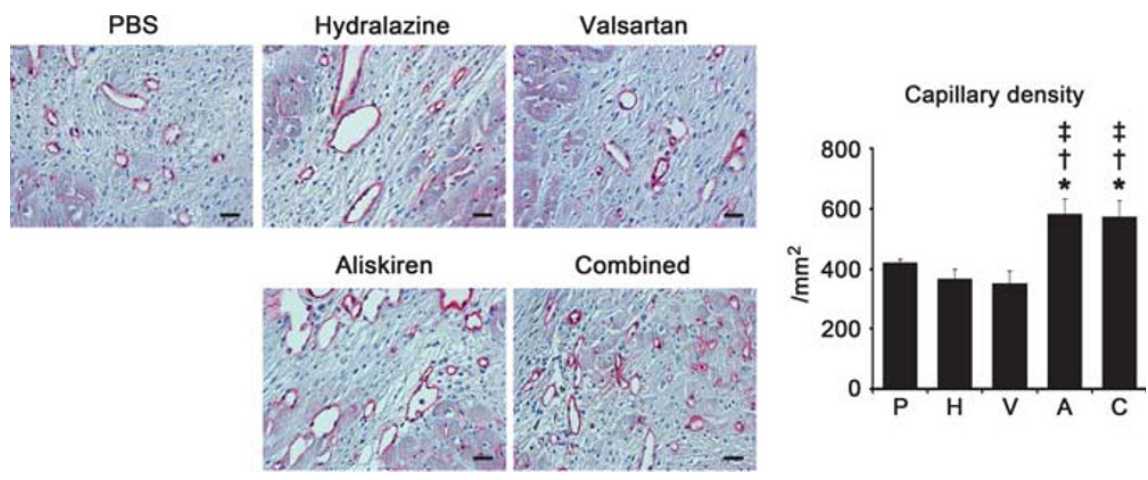

Figure 3 Capillary density in the peri-infarction area at 28 days after myocardial infarction. Capillary density was measured in specimens stained with anti-CD31 antibody ( $n=5$ for each group). P, the phosphate-buffered saline (PBS) group; $H$, the hydralazine group; V, the valsartan monotherapy group; $\mathrm{A}$, the aliskiren monotherapy group; $\mathrm{C}$, the combination therapy group. Scale bars $=20 \mu \mathrm{m}$. ${ }^{*} P<0.05$ vs. the PBS group; ${ }^{\dagger} P<0.05$ vs. the hydralazine group; ${ }^{\ddagger} P<0.05$ vs. the valsartan monotherapy group.

\section{Capillary density in the peri-infarction area}

The capillary density in the peri-infarction area at 28 days after MI was higher in the aliskiren group and the combination therapy group than in the PBS group, whereas the capillary density was not different between the PBS and the hydralazine groups (Figure 3). Interestingly, the capillary density in the peri-infarction area of the valsartan group was similar to that of the PBS group or the hydralazine group, and lower than the aliskiren group and the combination therapy group.
Oxidative stress in the peri-infarction and the non-infarcted area Lipid peroxidation assay demonstrated that at 28 days after MI, oxidative stress both in the peri-infarction area and in the noninfarcted area was ameliorated by valsartan or aliskiren monotherapy compared with PBS, whereas hydralazine did not reduce oxidative stress (Figure 4a). The combination therapy further reduced oxidative stress in the non-infarcted area than valsartan or aliskiren monotherapy, whereas oxidative stress in the peri-infarction area was 

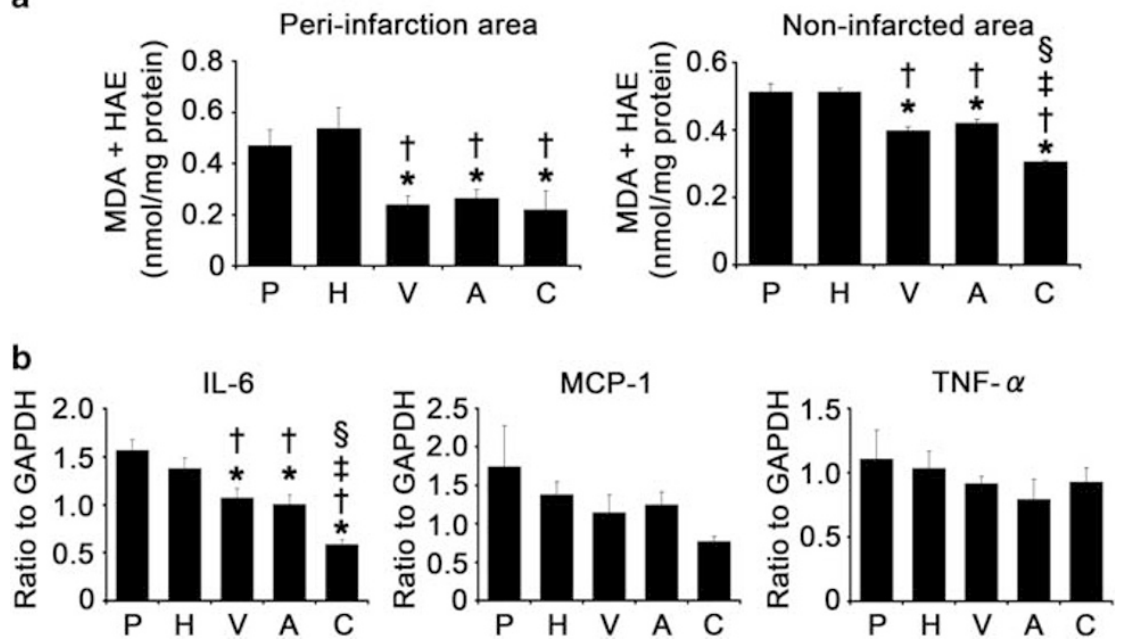

Figure 4 Oxidative stress and pro-inflammatory cytokine expression in the heart at 28 days after myocardial infarction (MI). (a) Oxidative stress in the peri-infarction and the non-infarcted area. Concentrations of malondialdehyde (MDA) and 4-hydroxyalkenals (HAE) were measured ( $n=4$ for each group). (b) Expression levels of interleukin-6 (IL-6), monocyte chemotactic protein-1 (MCP-1) and tumor necrosis factor- $\alpha$ (TNF- $\alpha$ ) in the non-infarcted area ( $n=5$ for each group). $\mathrm{P}$, the phosphate-buffered saline (PBS) group; $\mathrm{H}$, the hydralazine group; $\mathrm{V}$, the valsartan monotherapy group; $A$, the aliskiren monotherapy group; $\mathrm{C}$, the combination therapy group. ${ }^{*} P<0.05$ vs. the PBS group; ${ }^{\dagger} P<0.05$ vs. the hydralazine group; ${ }^{\ddagger} P<0.05$ vs. the valsartan monotherapy group; $\$ P<0.05$ vs. the aliskiren monotherapy group.

not different among the valsartan, the aliskiren and the combination therapy groups.

\section{Pro-inflammatory cytokine mRNA expression in the non-infarcted area}

At 28 days after MI, gene expression levels of IL- 6 in the non-infarcted area were lower in the valsartan and the aliskiren monotherapy groups than in the PBS group, whereas those in the hydralazine group was similar to those in the PBS group (Figure $4 \mathrm{~b}$ ). The dual therapy further suppressed IL- 6 expression in the non-infarcted area compared with either monotherapy. Monocyte chemotactic protein- 1 and tumor necrosis factor- $\alpha$ expression levels were not statistically different among the five groups, although the expression level of monocyte chemotactic protein-1 in the combination therapy group tended to be lower than that in the other four groups.

\section{DISCUSSION}

In this study, we showed that aliskiren ameliorated ventricular remodeling after MI similarly to valsartan at the doses of the same BP-lowering effect. At our tested doses, aliskiren improved survival rate after MI compared with PBS, whereas valsartan did not significantly reduce mortality because of cardiac rupture. We also found that the combination of valsartan and aliskiren exerted greater protective effects against ventricular remodeling after MI than either monotherapy, although additional BP-lowering effect was not observed with our tested doses.

Angiogenesis is very important for cardiac healing process after MI. In this study, we found that aliskiren promoted angiogenesis in periinfarction area, whereas valsartan did not enhance this process. Moreover, the addition of valsartan to aliskiren did not impair the beneficial effect of aliskiren. These results might explain why aliskiren and the dual therapy improved survival rate after MI compared with PBS or hydralazine, whereas valsartan did not reduce mortality. In the aliskiren and the combination therapy group, enhanced angiogenesis might lead to promotion of cardiac healing process after MI, and then to prevention of cardiac rupture. However, the reason why this difference occurred remains unclear. This may be attributable to the doses of drugs we used. Further studies will be needed to elucidate the mechanisms.

Oxidative stress and inflammation have been shown to be involved in the development of ventricular remodeling after MI. ${ }^{22-24}$ Oxidative stress and inflammation also depress cardiac function through impairment of $\mathrm{Ca}^{2+}$ homeostasis. ${ }^{23}$ The RAS is involved in the production of oxidative stress and inflammatory response. Angiotensin II stimulates nicotinamide adenine dinucleotide phosphate (NADPH) oxidase expression that induces the production of reactive oxygen species, and triggers inflammatory response. ${ }^{25-28}$ In this study, we found that aliskiren reduced oxidative stress and IL-6 expression in non-infarcted area similarly to valsartan with our tested doses. In addition, the combination of valsartan and aliskiren further suppressed oxidative stress and IL-6 expression in non-infarcted area than either monotherapy. These results may explain why the combination therapy ameliorated ventricular remodeling after MI compared with either monotherapy. As the combination therapy did not show further BPlowering effect, the synergistic effects might result from further suppression of tissue RAS.

In the previous clinical trials, the effect of the combination of ACEIs and ARBs has been tested on cardiovascular outcomes. ${ }^{29-33}$ However, this combination therapy has not resulted in the expected benefit possibly because of increases in both plasma renin activity and ACE escapes in addition to study-design issues. ${ }^{34}$ As DRIs decreases plasma renin activity, it was suggested that the combination of DRIs and ACEIs or ARBs might be the optimal combination of RAS-blocking agents. In fact, several animal studies and clinical trials showed the efficacy of this combination on cardiovascular outcomes. ${ }^{19,35-38}$

The effects of the combination of DRIs and ACEIs or ARBs on postMI outcomes were investigated in the ASPIRE (Aliskiren Study in Post-MI patients to Reduce rEmodelling) study. ${ }^{39}$ In this study, the addition of aliskiren to a standard optimal medical regimen, including an ACEI or an ARB, did not result in benefit with respect to ventricular remodeling compared to placebo and was associated with more adverse events in high-risk post-MI patients with $\mathrm{LV}$ 
systolic dysfunction, which is inconsistent with our results. In the ASPIRE study, patients were randomized at a median of 43 days after MI, and thus, in most of the enrolled subjects, aliskiren was added in the chronic phase after MI. The addition of aliskiren in the early post-MI period might have beneficial effect on post-MI outcomes. Moreover, in most of the enrolled patients, combined RAS-blocking agents were ACEIs and DRIs. Therefore, the efficacy of the combination of DRIs and ARBs might not be sufficiently evaluated. As ACE escape or angiotensin II production via non-ACE pathways could compromise the effect of ACEIs on the RAS, ${ }^{40-42}$ the combination of DRIs and ARBs might be beneficial.

There are several limitations in this study. This study is an animal study, and thus, our findings could not be simply extrapolated to human. Intense RAS blockade with dual therapy in the early phase after MI might not be tolerated in human, although it appears that this strategy was well tolerated in post-MI mice in this study. However, if dual therapy is well tolerated, the addition of aliskiren may be beneficial also in human.

In conclusion, our results indicate that aliskiren can be an alternative to ACEIs or ARBs in the treatment of post-MI patients. Moreover, the dual therapy of ARBs and DRIs may be more beneficial than either monotherapy. Further clinical trials will be warranted to sufficiently assess the safety and the efficacy of the use of aliskiren in post-MI patients.

\section{CONFLICT OF INTEREST}

The authors declare no conflict of interest.

\section{ACKNOWLEDGEMENTS}

Valsartan and aliskiren were kind gifts of Novartis. This study was supported in part by the Program for Promotion of Basic and Applied Researches for Innovations in Bio-Oriented Industry and by grants from the Ministry of Education, Culture, Sports, Science and Technology of Japan (Knowledge Cluster and New Research Area) and the Ministry of Health, Labor and Welfare of Japan.

1 Eisenberg MS, Mengert TJ. Cardiac resuscitation. N Engl J Med 2001; 344: 1304-1313.

2 Rogers WJ, Canto JG, Lambrew CT, Tiefenbrunn AJ, Kinkaid B, Shoultz DA, Frederick $\mathrm{PD}$, Every N. Temporal trends in the treatment of over 1.5 million patients with myocardial infarction in the US from 1990 through 1999: the National Registry of Myocardial Infarction 1, 2 and 3. J Am Coll Cardiol 2000; 36: 2056-2063.

3 Pfeffer MA, Braunwald E. Ventricular remodeling after myocardial infarction. Experimental observations and clinical implications. Circulation 1990; 81: 1161-1172.

4 Jessup M, Brozena S. Heart failure. N Engl J Med 2003; 348: 2007-2018.

5 White HD, Norris RM, Brown MA, Brandt PW, Whitlock RM, Wild CJ. Left ventricular end-systolic volume as the major determinant of survival after recovery from myocardial infarction. Circulation 1987; 76: 44-51.

6 Sun Y. Intracardiac renin-angiotensin system and myocardial repair/remodeling following infarction. J Mol Cell Cardiol 2010; 48: 483-489.

7 Pfeffer MA, Braunwald E, Moye LA, Basta L, Brown Jr EJ, Cuddy TE, Davis BR, Geltman EM, Goldman S, Flaker GC, Klein M, Lamas GA, Packer M, Rouleau J, Rouleau JL, Rutherford J, Wertheimer JH, Hawkins CM. Effect of captopril on mortality and morbidity in patients with left ventricular dysfunction after myocardial infarction. Results of the survival and ventricular enlargement trial. The SAVE Investigators. N Engl J Med 1992; 327: 669-677.

8 Yusuf S, Sleight P, Pogue J, Bosch J, Davies R, Dagenais G. Effects of an angiotensinconverting-enzyme inhibitor, ramipril, on cardiovascular events in high-risk patients. The Heart Outcomes Prevention Evaluation Study Investigators. N Engl J Med 2000; 342: $145-153$

9 Kober L, Torp-Pedersen C, Carlsen JE, Bagger H, Eliasen P, Lyngborg K, Videbaek J, Cole DS, Auclert L, Pauly NC. A clinical trial of the angiotensin-converting-enzyme inhibitor trandolapril in patients with left ventricular dysfunction after myocardial infarction. Trandolapril Cardiac Evaluation (TRACE) Study Group. N Engl J Med 1995; 333: 1670-1676.

10 Pfeffer MA, McMurray JJ, Velazquez EJ, Rouleau JL, Kober L, Maggioni AP, Solomon SD, Swedberg K, Van de Werf F, White H, Leimberger JD, Henis M, Edwards S,
Zelenkofske S, Sellers MA, Califf RM. Valsartan, captopril, or both in myocardial infarction complicated by heart failure, left ventricular dysfunction, or both. N Engl J Med 2003; 349: 1893-1906.

$11 \mathrm{Kim} \mathrm{S}$, Iwao H. Molecular and cellular mechanisms of angiotensin II-mediated cardiovascular and renal diseases. Pharmacol Rev 2000; 52: 11-34.

12 Brown MJ. Aliskiren. Circulation 2008; 118: 773-784.

13 Jensen C, Herold P, Brunner HR. Aliskiren: the first renin inhibitor for clinical treatment. Nat Rev Drug Discov 2008; 7: 399-410.

14 Westermann D, Riad A, Lettau O, Roks A, Sawatis K, Becher PM, Escher F, Jan Danser AH, Schultheiss HP, Tschope C. Renin inhibition improves cardiac function and remodeling after myocardial infarction independent of blood pressure. Hypertension 2008; 52: 1068-1075.

15 Katwa LC, Tyagi SC, Campbell SE, Lee SJ, Cicila GT, Weber KT. Valvular interstitial cells express angiotensinogen and cathepsin $\mathrm{D}$, and generate angiotensin peptides. Int J Biochem Cell Biol 1996; 28: 807-821.

16 Borges JC, Silva Jr JA, Gomes MA, Lomez ES, Leite KM, Araujo RC, Bader M, Pesquero JB, Pesquero JL. Tonin in rat heart with experimental hypertrophy. Am J Physiol Heart Circ Physiol 2003; 284: H2263-H2268.

17 The National Institutes of Health. Guide for the Care and Use of Laboratory Animals. Rev. 1985. edn. NIH publication, Bethesda.

18 Higashikuni Y, Sainz J, Nakamura K, Takaoka M, Enomoto S, Iwata H, Sahara M, Tanaka K, Koibuchi N, Ito S, Kusuhara H, Sugiyama Y, Hirata Y, Nagai R, Sata M. The ATP-binding cassette transporter BCRP1/ABCG2 plays a pivotal role in cardiac repair after myocardial infarction via modulation of microvascular endothelial cell survival and function. Arterioscler Thromb Vasc Biol 2010; 30: 2128-2135.

19 Yamamoto E, Kataoka K, Dong YF, Nakamura T, Fukuda M, Tokutomi Y, Matsuba S, Nako H, Nakagata N, Kaneko T, Ogawa H, Kim-Mitsuyama S. Aliskiren enhances the protective effects of valsartan against cardiovascular and renal injury in endothelial nitric oxide synthase-deficient mice. Hypertension 2009; 54: 633-638.

20 Ino J, Kojima C, Osaka M, Nitta K, Yoshida M. Dynamic observation of mechanicallyinjured mouse femoral artery reveals an antiinflammatory effect of renin inhibitor. Arterioscler Thromb Vasc Biol 2009; 29: 1858-1863.

21 Nakamura K, Sata M, Iwata H, Sakai Y, Hirata Y, Kugiyama K, Nagai R. A synthetic small molecule, ONO-1301, enhances endogenous growth factor expression and augments angiogenesis in the ischaemic heart. Clin Sci (Lond) 2007; 112: 607-616.

22 Sun Y. Myocardial repair/remodelling following infarction: roles of local factors. Cardiovasc Res 2009; 81: 482-490.

23 Hori M, Nishida K. Oxidative stress and left ventricular remodelling after myocardial infarction. Cardiovasc Res 2009; 81: 457-464.

24 Giordano FJ. Oxygen, oxidative stress, hypoxia, and heart failure. J Clin Invest 2005; 115: 500-508.

$25 \mathrm{Lu} \mathrm{L}$, Quinn MT, Sun Y. Oxidative stress in the infarcted heart: role of de novo angiotensin II production. Biochem Biophys Res Commun 2004; 325: 943-951.

26 Bataller R, Schwabe RF, Choi YH, Yang L, Paik YH, Lindquist J, Qian T, Schoonhover R, Hagedorn $\mathrm{CH}$, Lemasters JJ, Brenner DA. NADPH oxidase signal transduces angiotensin II in hepatic stellate cells and is critical in hepatic fibrosis. J Clin Invest 2003; 112: 1383-1394.

27 Touyz RM, Tabet F, Schiffrin EL. Redox-dependent signalling by angiotensin II and vascular remodelling in hypertension. Clin Exp Pharmacol Physiol 2003; 30: 860-866.

28 Gill PS, Wilcox CS. NADPH oxidases in the kidney. Antioxid Redox Signal 2006; 8: 1597-1607

29 White HD, Aylward PE, Huang Z, Dalby AJ, Weaver WD, Barvik S, Marin-Neto JA, Murin J, Nordlander RO, van Gilst WH, Zannad F, McMurray JJ, Califf RM, Pfeffer MA. Mortality and morbidity remain high despite captopril and/or Valsartan therapy in elderly patients with left ventricular systolic dysfunction, heart failure, or both after acute myocardial infarction: results from the Valsartan in Acute Myocardial Infarction Trial (VALIANT). Circulation 2005; 112: 3391-3399.

30 Phillips CO, Kashani A, Ko DK, Francis G, Krumholz HM. Adverse effects of combination angiotensin II receptor blockers plus angiotensin-converting enzyme inhibitors for left ventricular dysfunction: a quantitative review of data from randomized clinical trials. Arch Intern Med 2007; 167: 1930-1936.

31 Yusuf S, Teo KK, Pogue J, Dyal L, Copland I, Schumacher H, Dagenais G, Sleight P, Anderson C. Telmisartan, ramipril, or both in patients at high risk for vascular events. N Engl J Med 2008; 358: 1547-1559.

32 Mann JF, Schmieder RE, McQueen M, Dyal L, Schumacher H, Pogue J, Wang X, Maggioni A, Budaj A, Chaithiraphan S, Dickstein K, Keltai M, Metsarinne K, Oto A, Parkhomenko A, Piegas LS, Svendsen TL, Teo KK, Yusuf S. Renal outcomes with telmisartan, ramipril, or both, in people at high vascular risk (the ONTARGET study): a multicentre, randomised, double-blind, controlled trial. Lancet 2008; 372: 547-553.

33 Lakhdar R, Al-Mallah MH, Lanfear DE. Safety and tolerability of angiotensin-converting enzyme inhibitor versus the combination of angiotensin-converting enzyme inhibitor and angiotensin receptor blocker in patients with left ventricular dysfunction: a systematic review and meta-analysis of randomized controlled trials. J Card Fail 2008; 14: 181-188.

34 Weber MA, Giles TD. Inhibiting the renin-angiotensin system to prevent cardiovascular diseases: do we need a more comprehensive strategy? Rev Cardiovasc Med 2006; 7: 45-54.

35 Parving $\mathrm{HH}$, Persson F, Lewis JB, Lewis EJ, Hollenberg NK. Aliskiren combined with losartan in type 2 diabetes and nephropathy. N Engl J Med 2008; 358 : 2433-2446 
36 Persson F, Rossing P, Reinhard H, Juhl T, Stehouwer CD, Schalkwijk C, Danser AH, Boomsma F, Frandsen E, Parving $\mathrm{HH}$. Renal effects of aliskiren compared with and in combination with irbesartan in patients with type 2 diabetes, hypertension, and albuminuria. Diabetes Care 2009; 32: 1873-1879.

37 Solomon SD, Appelbaum E, Manning WJ, Verma A, Berglund T, Lukashevich V, Cherif Papst C, Smith BA, Dahlof B. Effect of the direct renin inhibitor aliskiren, the Angiotensin receptor blocker losartan, or both on left ventricular mass in patients with hypertension and left ventricular hypertrophy. Circulation 2009; 119: 530-537.

38 McMurray JJ, Pitt B, Latini R, Maggioni AP, Solomon SD, Keefe DL, Ford J, Verma A, Lewsey J. Effects of the oral direct renin inhibitor aliskiren in patients with symptomatic heart failure. Circ Heart Fail 2008; 1: 17-24.
39 Solomon SD, Hee Shin S, Shah A, Skali H, Desai A, Kober L, Maggioni AP, Rouleau JL, Kelly RY, Hester A, McMurray JJ, Pfeffer MA. Effect of the direct renin inhibitor aliskiren on left ventricular remodelling following myocardial infarction with systolic dysfunction. Eur Heart J 2011; 32: 1227-1234.

40 Hollenberg NK, Fisher ND, Price DA. Pathways for angiotensin II generation in intact human tissue: evidence from comparative pharmacological interruption of the renin system. Hypertension 1998; 32: 387-392.

41 Ihara M, Urata H, Kinoshita A, Suzumiya J, Sasaguri M, Kikuchi M, Ideishi M, Arakawa K. Increased chymase-dependent angiotensin II formation in human atherosclerotic aorta. Hypertension 1999; 33: 1399-1405.

42 Wong J, Patel RA, Kowey PR. The clinical use of angiotensin-converting enzyme inhibitors. Prog Cardiovasc Dis 2004; 47: 116-130. 